\title{
THE ROLE OF BIM FOR SAFETY AND SECURITY MANAGEMENT
}

\author{
FABIO GARZIA ${ }^{1,2,3}$ \& MARA LOMBARDI ${ }^{1}$ \\ ${ }^{1}$ Safety \& Security Engineering Group - DICMA, SAPIENZA - University of Rome, Italy \\ ${ }^{2}$ Wessex Institute of Technology, Southampton, United Kingdom \\ ${ }^{3}$ European Academy of Sciences and Arts, Salzburg, Austria
}

\begin{abstract}
A vital component of any type of organization for the prevention of incidental events and/or voluntary attacks against people and tangible and intangible assets as well as for their protection when incidental events and/or voluntary attacks happen is represented by safety and security management. Because of the incessant development of new dangers and threats, safety and security management requests unceasing updating, by means of powerful and flexible tools, such as BIM, which must be integrated via a multidisciplinary approach, considering also economic features that must optimized from the cost/benefit point of view. Integrated technological systems represent efficient tools to create solutions than can support safety and security management in a competent manner, considering also budgets optimization. For this reason, it is necessary to utilize a wide-range approach which, thanks to its flexibility, allows for the realization of an integrated multidisciplinary model for safety and security management (IMMSSM) which can be supported by a suitable Integrated Technological System Framework (ITSF), even based on Internet of Everything (IoE). To achieve an operative IMMSSM, it is necessary to optimize the available tools from the cost/benefit point of view. This goal represents a difficult challenge because, in general, restricted funds are available. From this point of view, efficient and flexible tools, such as BIM, represent vital elements to obtain solutions characterized by an optimal cost/benefit ratio. The purpose of this paper is to illustrate the role of BIM for safety and security management, showing its peculiar features from this point of view and its versatility both for the realization of resourceful IMMSSM and related ITSF, even based on IoE.

Keywords: BIM, Genetic Algorithms, Internet of Everything, Internet of Things, IoE integrated system, safety management, security management.
\end{abstract}

\section{INTRODUCTION}

Safety and security management represent a significant and powerful tool for any sort of organization for the prevention of incidental events (fires, floods, hurricanes, earthquakes, etc.) and/or voluntary attacks (vandalism, thefts, espionage, etc.) against people and tangible and intangible assets as well as for their protection when incidental events and/or voluntary attacks happen. When an incidental event and/or a voluntary attack happen, it is essential to moderate it during the initial phase and during the following phases, by means of essential tools represented by emergency management, business/service continuity and disaster recovery.

Because of the constant unwanted development of novel dangers and threats, safety and security management needs continuous updating, using resourceful tools, such as BIM, which must be integrated via a multidisciplinary approach, keeping into consideration financial aspects that must optimized from the cost/benefit point of view.

BIM represents a collaboration software which offers a source for each discipline to add digital, facility-specific knowledge into a single shared dynamic model, typically online. The most evident characteristic is represented by its capability of developing 3D drawings. This type of representation simplifies the process of understanding how a complex site or a completed building will look, how people will move around and how spaces are reciprocally related, providing vital information for safety and security management. 
Anyway, BIM is more than that a simple tool to develop 3D drawings. In fact, conventional $3 \mathrm{D}$ drawings illustrate a facility in length, width, and height, but with BIM there is also 4D (represented by time), 5D (represented by cost) and 6D (represented by life cycle) information that represent, once more, vital data for safety and security management.

From this point of view, BIM can be used for detailed programming and design work since it allows for conceptualize, plan, schedule, estimate, coordinate, verify and do "what if' analyses, etc.

Regarding safety aspects, BIM is very useful for construction safety, maintenance safety, emergency planning and management, occupational safety planning, safety analysis, fire engineering and a plenty of other aspects [1-3].

Regarding security aspects, BIM is very useful for [4-7]:

1. Security design - conduit and conductor routing and sizing and device placement, especially intrusion detection, access control, CCTV.

2. On-line data storage - rapid retrieval and analysis, especially digital video images.

3. Facility programming, concept development and planning - mapping circulation, pedestrian and vehicular; Crime Prevention Through Environmental Design (CPTED) design; space utilization cost/benefit studies (such as squared-meter or square-foot costs).

4. Tracking equipment and materials - ordering on line.

5. Cost analyses - construction cost estimates and take-offs (quantity counts); cost-benefit analyses that justify operating costs of the security department and estimate annual losses and shrinkage.

6. Building/fire/life-safety code review - automated code analyses.

7. Standardizing security specifications - assembling pertinent specifications.

8. International Organizations for Standardization (ISO) standards compliance and certification.

9. Automated security standards and guidelines review.

10. Resolve security and life-safety code conflicts.

11. Moving the security control centre during each phase; Construction scheduling -materials delivered on a 'just-at-the-right-time' basis rather than stored on site.

12. Life-cycle costs calculations for security operations since the design and construction of a building is typically only 2 to $5 \%$ of 20 -year operating costs. Thus, a design that reduces annual security operating costs can result in remarkable savings.

13. Coordinating design disciplines thanks to the BIM 'clash detection' feature since safety, security, emergency, electrical, mechanical etc. drawings are complex and difficult to coordinate in the realization phase. From this point of view, BIM can discover these conflicts before the beginning of constructive activities.

14. What-If Analysis since BIM could calculate and compare the installing costs and operating costs of standard intrusion detection sensors, with respect to different coverage devices and CCTV cameras, as compared to megapixel cameras and determine which option is the most cost-effective.

15. Ongoing Operations: schedule security preventive maintenance and optimize guard postings, in particular guard tours, on a continual real-time basis.

Due to the limited space, the aspects illustrated above represents only a subgroup of what BIM can ensure in safety and security management, in particular way from the integrated point of view which ensures that a certain BIM application is valid both for safety and 
security at the same time, providing valuable solutions from the cost/benefit point of view. An example is represented by the integration and synergy deriving from the joint use of BIM and BMS (Building Management System) which can be also integrated with Internet of Things (IoT)/Internet of Everything (IoE). Further, other significant aspects and application will be surely discovered in future with its growing and intensive usage.

BIM is therefore also extremely important for the typical study, designed, realization and maintenance phases of integrated technological systems [8-12] which represent efficient tools to provide solutions capable of supporting safety and security management in a resourceful way, in particular when Internet of Everything is used, which allow people, things (mobile terminals, devices, actuators, smart sensors, wearable devices, etc.), data/information/knowledge and procedures to be properly connected to achieve the desired purposes [13, 14].

Due to this motive, it is essential to utilize a wide-range approach which lets the realization of an integrated multidisciplinary model for safety and security management (IMMSSM) [15], thanks to its flexibility. The IMMSM can be efficiently supported by a proper Integrated Technological System Framework (ITSF), even based on Internet of Everything (IoE-ITSF) $[13,14]$.

To provide an effective IMMSSM, it is necessary to optimize the available tools from the cost/benefit point of view

This aim represents a hard task since reduced funds are generally available, and it is necessary to utilize them in the most efficient way, achieving the maximum reduction of damages due to different threats and the best management of residual risks via emergency management, service/ business continuity and disaster management.

From this point of view, powerful and flexible tools, such as BIM, represent vital elements to obtain solutions characterized by an optimal cost/benefit ratio [1-7].

The purpose of this paper is to illustrate the role of BIM for safety and security management, showing its peculiar features from this point of view and its versatility both for the realization of efficient IMMSSM and related ITSF, even based on IoE (IoE-ITSF).

In the following, the already existing research [15] is briefly illustrated, due to the limited space available, and then the great advantages deriving from the use of BIM to the already existing research is studied and demonstrated from the quantitative point of view.

\section{THE INTEGRATED MULTIDISCIPLINARY MODEL FOR SAFETY AND SECURITY MANAGEMENT}

Because safety and security deal with risks, it is vital to have a general description of it for our goals. Therefore, risk R can be defined as the probability $\mathrm{P}$ of a quantifiable damage, injury, liability, loss, or any other negative occurrence (briefly indicated with damage $\mathrm{D}$, as a function of the considered context) which is provoked by external or internal vulnerabilities. The risk $\mathrm{R}$ can therefore be defined as:

$$
R=f(P, D)
$$

where $\mathrm{f}(*)$ represents a proper function, which depends on the specific context, P represents the probability of the risk, variable between 0 and 1 , and $\mathrm{D}$ represents the damage which can be defined according to a chosen reference scale, as a function of the considered organization. Without loss of generality and to keep a general approach, we suppose the damage D to be variable between 0 and 10 in the considered context.

The different activities (risk analysis, impact analysis and evaluation of the derived damages, risks and damages mitigation, residual risks management via emergency management, service/ 
business continuity and disaster recovery) and the associated operative tools (OTs) required for safety and security management must be suitably systematized in a general model which could be useful in most contexts for most organizations, to achieve optimal and desired results.

Due to this motive, a suitable integrated multidisciplinary model for safety and security management (IMMSSM) has already been studied [15], representing a general model valuable for most organizations. The scheme of IMMSSM is shown in Figure 1.

Therefore, considering a general organization, all the essential actions have been suitably associated according to the proposed general scheme shown in Figure1 that starts with a proper risk analysis [16-19].

The prevention/protection activities can be done using wide-range and multidisciplinary operative factors or tools (OTs), that are represented by: countermeasures (CM) ([8-14, 20]), security policies and procedures (briefly, procedures or PR), human factors and resources (briefly, human factor or HF) [21].

Each of these OT is characterized by proper expenses which have to be considered in the creation of the prevention/protection doings.

Residual risk mitigation can be executed via essential tools, aided by OTs, which are represented by emergency management (EM), Business/service continuity (BSC) Disaster recovery (DR) which can also be properly realized via the above OTs and considering the associated costs. Specific OTs can also be advantageous at the same time not only for prevention and protection in risks mitigation but also for emergency management, business and service continuity, and disaster recovery. A typical OT of this type is represented by CCTV in each area but also BIM, as it is demonstrated in the following, represents a powerful tool capable of greatly enhancing the flexibility of OTs. A suitable Integrated Technological System Framework (ITSF) aided by a proper optimization procedure for the use of OTs from the cost/benefit point of view, is capable of reducing the general risk of the organization at minimum cost, as is shown below, thus guaranteeing the optimal implementation of the IMMSSM at minimum cost, according to the wished goals.

All the elements of the IMMSSM illustrated above interact mutually: if there is a variation in one of them, such as a new threat to face, the related change of risk analysis produces an inevitable modification in all the other elements, since the model is stringently related.

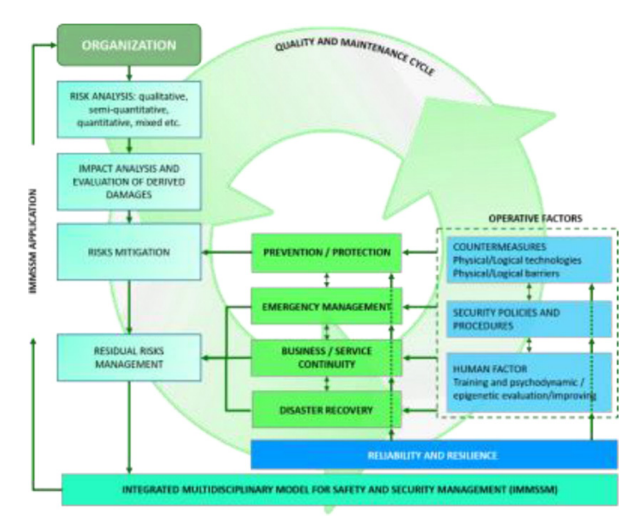

Figure 1: Scheme of the Integrated Multidisciplinary Model for Safety and Security Management (IMMSSM). 
Reliability and resilience represent fundamental elements to be considered for OTs, emergency management, business /service continuity and disaster recovery, as indicated in Figure 1.

The IMMSSM, because of the variety of features, analyses and procedures that must be considered in normal and critical situations, unavoidably requests an Integrated Technological System Framework (ITSF) for its sustenance and for the actuation of all the strategies and procedures.

The analysis, planning and management of the maintenance and quality of model and of the associated supportive integrated technological system, as well as the initial realization cost and annual cost, must also be considered for the realization of the IMMSSM.

To realize a high-performance IMMSSM, it is necessary to optimize the usage of OTs from a cost/benefit point of view, as shown in the following, considering not only the cost of initial execution but also the annual costs.

From this point of view, the great advantages derived by using BIM in the above IMMSSM and related ITSF are demonstrated in the following.

\section{DESCRIPTION OF THE INTEGRATED TECHNOLOGICAL SYSTEM FRAMEWORK}

The realization of a suitable Integrated Technological System Framework based on Internet of Everything (IoE-ITSF) is strongly recommended to support the IMMSSM, so that it is possible to ensure the whole goals of the IMMSSM with high flexibility and modularity, letting possible at any time to translate any eventual adjustment of the IMMSSM into a fast and low-cost adjustment of the related IoE-ITSF.

This object can be achieved via integrated systems [8-12] and innovative technologies, represented by Internet of Everything ( $\mathrm{IoE}$ ), which can join people, things (mobile terminals, devices, actuators, smart sensors, wearable devices, etc.), data/information/knowledge and procedures to achieve the whished objective $[13,14]$.

In Figure 2, the general scheme of the proposed IoE-ITSF is shown.

The IoE-ITSF is characterized by a high modularity which allows for the addition at any time of any device, element, system etc. which needs to be integrated in the IoE system.

Since the IoE-ITSF is intended to represent a universal framework adapt for the most organizations which can have the presence of external visitor's, for security reason the networks used to achieve

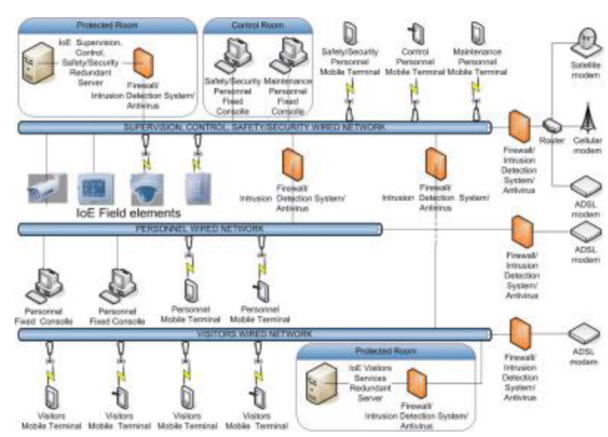

Figure 2: Scheme of the Integrated Technological System Framework based on Internet of Everything (IoE-ITSF) for the sustenance of the Integrated Multidisciplinary Model for Safety and Security Management (IMMSSM). 
supervision, control and safety/security services, internal personnel services and visitor's services are properly detached by physical and logical points of view.

The IoE-ITSF can communicate with all the 'IoE objects', signalling any hazardous or dangerous condition to the operators (personnel in the control room, security personnel, safety personnel, maintenance personnel, Police, Fire Brigades, Civil Protection, Medical staff, etc.), in real time, via any type of communication means.

Further information related to the IoE-ITSF can be found in the related reference [15].

\section{EFFECT OF BIM ON THE IMPLEMENTATION OF THE INTEGRATED \\ MULTIDISCIPLINARY MODEL FOR SAFETY AND SECURITY MANAGEMENT}

To make available an operative IMMSSM, it is required, from a cost/benefit point of view, to adjust the existing operative tools (OTs), in view of also the role of BIM, that represents the scope of the paper.

This is a quite difficult objective to be achieved because of the limited funds generally available. For this reason, it is necessary its usage in the most effective mode, to guarantee the maximum reduction of damages due to different threats and the best management of residual risk via emergency management, service/business continuity and disaster management.

This is a typical nonlinear multi-parameter and multi-goals problem that has already been resolved proficiently using Genetic Algorithms (GAs) [15] without keeping into consideration the role of BIM which is studied in the following after briefly illustrating, due to the limited space available, the results already obtained within the previous research [15].

From this point of view, a proper implementation technique based on Genetic Algorithms (GAs) has been studied. It can optimize the usage of the available operative tools (OTs) and can set up an IMMSSM fitted to the operative context and skilled to guarantee the best performances with the best cost/benefit ratio [15].

The ad-hoc GA has been studied and realized so that, once individuated and evaluated all the threats by means of risk analysis, and all the available OTs, together with the relative realization cost and annual maintenance costs (to keep into consideration the total cost of the useful vital cycle of them), it is capable, given a certain realization budget, to find the best combination of OTs, between the available ones, that can be used to ensure the maximum reduction of total risk [15].

From this point of view, after a proper preliminary and detailed analysis that allows to create a series of arrays represented by RA (Risks Array), P (Probability Array), D (Damage Array), CM (Countermeasures Array), Procedures Array (PR), Human Factor Array (HF), EM (Emergency Management Array), BSC (Business and Service Continuity), DR containing all the information necessary to the GA to perform its optimization role [15].

The purpose of the optimization is synthetized by a proper fitness function which drives the evolution of the population of the GA to reach the desired final goal. The ad-hoc GA is not illustrated here for brevity. The GA has been tested on more than 800 real and random situations, to attain, as much as possible, general mean results relevant for any type of situation. All the results are got with quite quick converge. Because of the great volume of final data attained and because of the quantity of results that can be taken out from this great volume of final data, only the most noteworthy outcomes are showed in the following, due to the limited space available.

A significant parameter to be considered in mining noteworthy information is represented by the mean OT flexibility MOT $_{\text {flexibility }}$, represented by the mean value, extended over all the OTs, of the number of risks of the P, D, EM, BSC, DR arrays where each OT is used, and the 
total number of risks of the same arrays. The $\mathrm{MOT}_{\text {flexibility }}$ expresses, therefore, the mean flexibility of OTs and, as defined, can vary between 1 (all OTs can be used for any activities) and 0 (not any OT can be used for any activities, which is a not real situation). A very flexible OT is, for example, represented by CCTV which can be utilized, in given zones and in given situations, both to reduce the probability and damage of a given risk but can be also be advantageous for emergency management, business and service continuity and for disaster recovery. It is obvious that the greater $\mathrm{MOT}_{\text {flexibility }}$ and the greater the GA possibility of optimization, as will be shown in the following.

Another significant parameter is represented by the investment ratio IR, represented by the ratio between the investment cost, represented by the sum of the realization cost and the annual cost, and the total cost of OTs. The IR expressed can vary between 1 (all OTs are used for any activity since the investment allows it) and 0 (not any OT can be used for any activity, since the investment does not allow, which is a not real situation). It is obvious that the greater IR and the greater the GA possibility of optimization, as will be shown in the following.

In this part, BIM contribute is not considered, to demonstrate its powerful contribution later, when it is properly considered, demonstrating all its positive influence due to its great flexibility and usefulness.

It is also evident that if all the possible OTs (excluding BIM which is the goal of this study and whose effect is studied later) are used, the total risk, characterised by the sum of all considered risks, is reduced at the initially minimum planned level (that is a reduction value equal to $100 \%$ ) while if not any OT is used, the total risk remains at the initially maximum planned level (that is a reduction value equal to $0 \%$ ). The total risk reduction (RR) expressed as a percentage, can represent a suitable parameter to measure the optimization abilities of the GA.

The total risk reduction RR, expressed in percentage form, as a function of investment ratio IR, for different values of MOT flexibility. $_{\text {, is shown in Figure } 3 .}$

Figure 3 shows how the GA is capable of reducing, in an efficient way, the RR as a function of both IR and MOT flexibility, as expected [15]. It is obvious that as IR increases, more OTs can be used by GA for risk reduction, and the curves grow, according to different profiles, as a function of $\mathrm{MOT}_{\text {flexibility. }}$

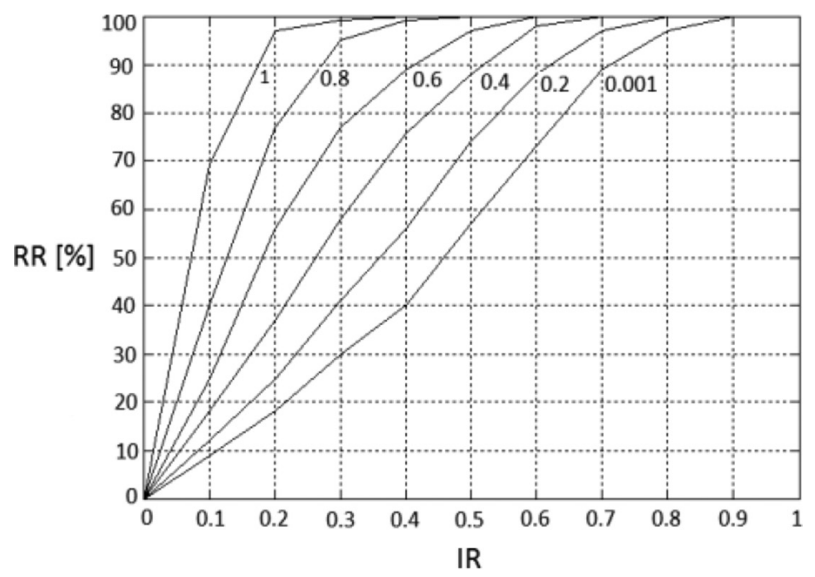

Figure 3: Total risk reduction (\%) as a function of number of investment ratio IR for different values of MOT $_{\text {flexibility }}$. 
It is also evident that the greater the MOT flexibility then the greater the GA possibility of optimization. When $\mathrm{MOT}_{\text {flexibility }}$ tends to 1 (maximum value reachable) the OTs can be used in most activities and this allows the GA to perform its maximum optimization capabilities, reaching total risk reduction of $100 \%$ with investment ratio equal to about 0.4 . When $\mathrm{MOT}_{\text {flexibility }}$ tends to 0 (minimum theoretical reachable value), the OTs cannot be used in most activities and this does not permit the GA to best perform its optimization abilities, reaching a total risk reduction of $100 \%$ with investment ratio IR equal to about 0.9 . Even in this worst case, anyway, the GA can ensure a decrease of IR. In Figure 3, it is not considered the situation $\mathrm{MOT}_{\text {flexibility }}=0$ since this situation is unreal. For this reason, only the situation when $\mathrm{MOT}_{\text {flexibility }}=0.001$ is considered, as lower values of $\mathrm{MOT}_{\text {flexibility }}$ tend to produce curves that are practically superimposed to this last curve.

It is therefore evident from the results of the previous research illustrated synthetically above, that the more the $\mathrm{MOT}_{\text {flexibility }}$ is close to one and the more the final solution is characterized by a final solution which is extremely efficient from the cost/benefit point of view [15].

The number of generations necessary to the GA to reach the final optimal solution represents a very important parameter, together with the initial population, since it gives an indication of the computation charge that, once related to the computation resources available, provides an exact value of the time necessary to reach the desired final optimal solution.

In Figure 4, the number of generations necessary to the GA to reach the final optimal solution as a function of investment ratio IR, for different values of MOT $_{\text {flexibility }}$ is shown.

As it is possible to see from Figure 4, the number of generations necessary to reach the final solution grows with the investment ratio since the GA has to work more due to the fact

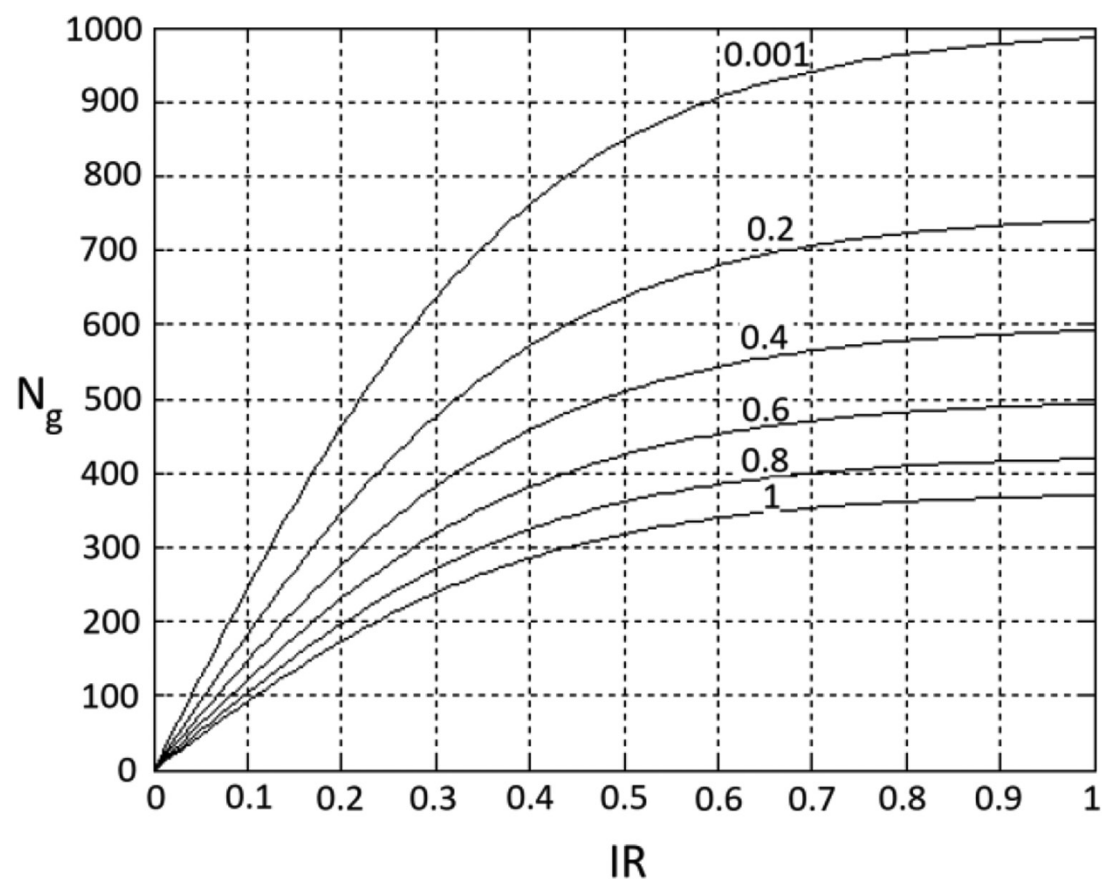

Figure 4: Number of generations necessary to the GA to reach the final optimal solution as a function of investment ratio IR, for different values of $\mathrm{MOT}_{\text {flexibility }}$. 
that the greater IR and the greater is the number of OTs available which can be used to find a final optimal solution.

For lower values of $\mathrm{MOT}_{\text {flexibility }}\left(\mathrm{MOT}_{\text {flexibility }}=0.001\right)$ due to the reduced flexibility of OTs, the GA must work more to reach the final optimal solutions and, for this reason, the number of generations is greater.

For greater values of $\mathrm{MOT}_{\text {flexibility }}$, $\left(\mathrm{MOT}_{\text {flexibility }}=1\right)$ due to the great flexibility of OTs, the GA must work less to reach the final optimal solutions and, for this reason, the number of generations is lesser.

It is now important to investigate the role of BIM, to verify if and how it is capable to contribute in an efficient way, under all the point of view, to the safety and security management, which represents the scope of the paper.

As demonstrated in the following, BIM is capable of ensuring new low cost solutions and of integrating and powering the existing ones, increasing the $\mathrm{MOT}_{\text {flexibility }}$ and optimizing the cost/benefit ration of the final solutions for safety and security management.

A significant and synthetic parameter useful to verify the effect of BIM use is represented by the $\mathrm{MOT}_{\text {flexibility, }}$, since it greatly influences the capability of reaching the final goal in an efficient way, as shown in Figure 3.

From this point of view, all the possible uses of BIM, partially indicated above, have been individuated and normalized in a scale varying from 0 (not any use of BIM) to $100 \%$ (all the possible and individuated uses of BIM are made) and the MOT $_{\text {flexibility }}$ has been calculated as a function of BIM use (expressed in percentage).

In Figure 5, the $\mathrm{MOT}_{\text {flexibility }}$ as a function of BIM use (expressed in percentage), for different values of initial $\mathrm{MOT}_{\text {flexibility }}(0.001,0.2,0.4,0.6,0.8)$ is shown. It gives a clear and comparative idea of both the scenarios regarding IMMSSM with and without BIM.

As it is possible to see from Figure 5, the introduction of BIM can increase the MOT flexibility, $_{\text {, }}$ for any kind of initial value of MOT $_{\text {flexibility }}{ }^{*}$

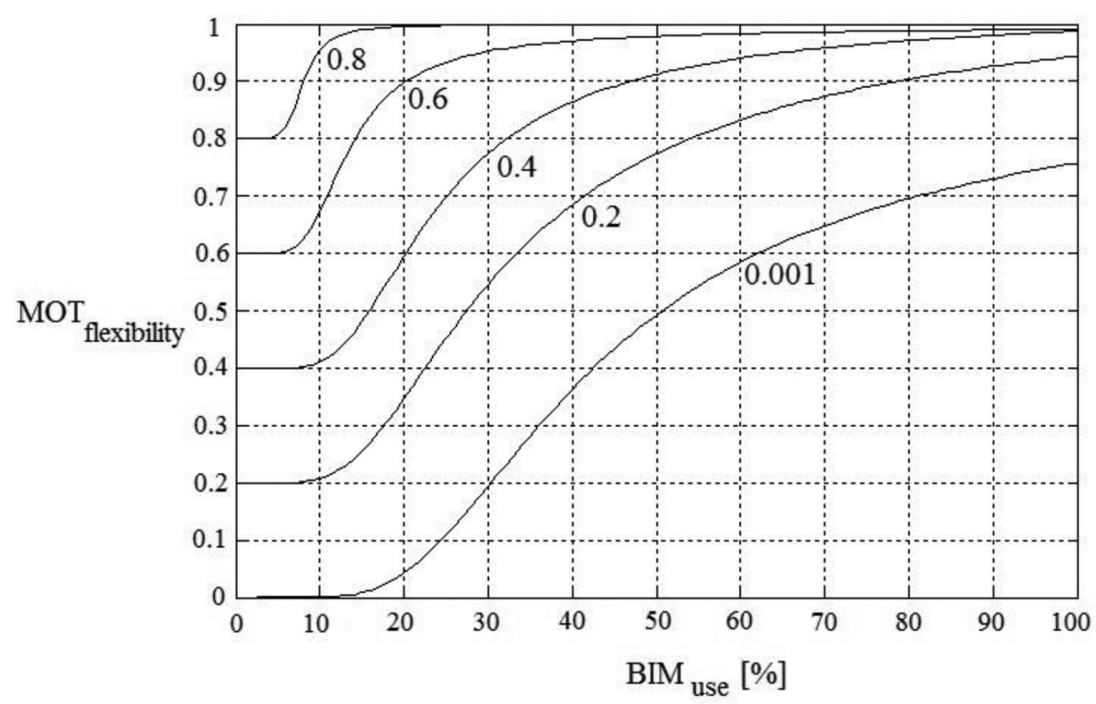

Figure 5: $\mathrm{MOT}_{\text {flexibility }}$ as a function level of $\mathrm{BIM}_{\text {use, }}$, expressed in percentage, for different values of initial $\mathrm{MOT}_{\text {flexibility }}(0.001,0.2,0.4,0.6,0.8)$. 
Since the RR illustrated in Figure 3 has been considered for four significant values of $\mathrm{MOT}_{\text {flexibility }}(0.001,0.2,0.4,0.6,0.8)$, the same was made in Figure 5.

Results shown in Figure 5 demonstrate that when not any BIM usage is done $\left(\mathrm{BIM}_{\text {use }}=0 \%\right)$, the $\mathrm{MOT}_{\text {flexibility }}$ remains obviously the same for the four considered initial values $(0.001,0.2,0.4,0.6$, 0.8). As soon as BIM use increases, due to its versatility and its power, the $\mathrm{MOT}_{\text {flexibility }}$ grows in a significant way for lower initial values of the MOT $_{\text {flexibility }}$ itself $(0.001)$ and in a less significant way for greater initial values of the MOT $_{\text {flexibility }}$ itself (0.8).

When BIM is integrally used $\left(\mathrm{BIM}_{\text {use }}=100 \%\right)$, the MOT flexibility $_{\text {reaches the maximum }}$ level for the four considered initial values $(0.001,0.2,0.4,0.6,0.8)$. In particular, for greater initial values of $\mathrm{MOT}_{\text {flexibility }}$, it is possible to reach a final value of $\mathrm{MOT}_{\text {flexibility }}$ close to 1 (maximum flexibility of OTs and therefore reaching of the minimum level of risk with the lowest possible cost) with a quite limited use of BIM.

It is also evident that, due to the reduced cost of BIM usage, its introduction allows to greatly increase the cost/benefit ratio, reaching high-performing solutions, from the safety and security management point of view, with reduced costs with respect to solutions which do not use it.

Since the number of generations necessary to the GA to reach the final optimal solution represents a very important parameter, as illustrated before, it is important to evaluate this aspect considering the contribute of BIM usage, comparing it with the previous results where BIM is not used.

In Figure 6, the number of generations necessary to the GA to reach the final optimal solution as a function investment ratio IR when $\mathrm{MOT}_{\text {flexibility }}=0.001$, for different values $\mathrm{BIM}_{\text {use, }}$, is shown.

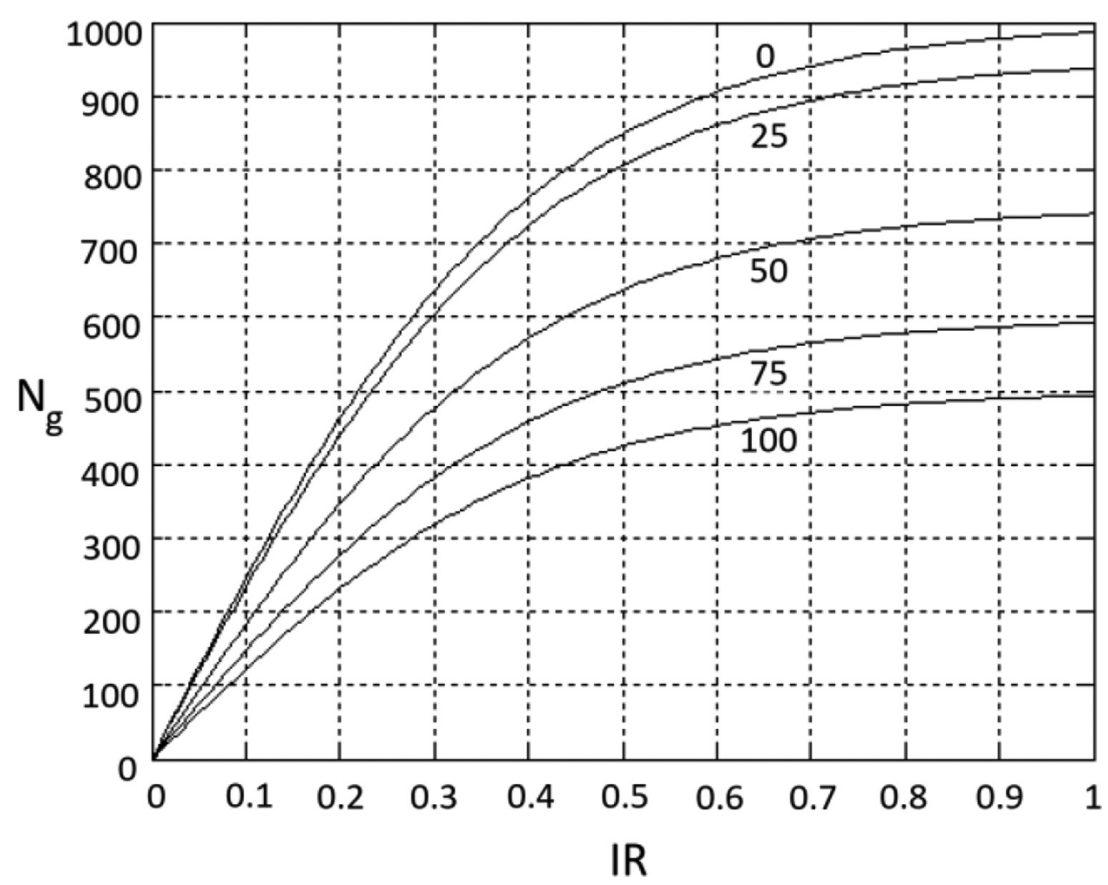

Figure 6: Number of generations necessary to the GA to reach the final optimal solution as a function investment ratio IR, when $\mathrm{MOT}_{\text {flexibility }}=0.001$, for different values $\mathrm{BIM}_{\text {use }}$ expressed in percentage. 
In Figure 6, only the worst situation of $\mathrm{MOT}_{\text {flexibility }}=0.001$ has been illustrated since the situation obtained for the other considered values $(0.2,0.4,0.6,0.8,1)$ is similar, a part the absolute values that obviously vary as a function of MOT $_{\text {flexibility }}$ and for this reason, the related figures are not shown here due to the limited space available.

In this case, it is possible to see the great advantages deriving for BIM usage also in term of reduction of number of generations necessary to reach the final optimal solution since, even if the same curve related to $\mathrm{MOT}_{\text {flexibility }}=0.001$ is used, the number of generation reduces when BIM usage increases.

As it is possible to see from Figure 6, the number of generations necessary to reach the final solution grows with the investment ratio since the GA has to work more due to the fact that the greater IR and the greater is the number of OTs available which can be used to find a final optimal solution. The curve related to BIM usage equal to zero is obviously the same curve present in Figure 4.

For lower values of BIM usage, (BIM usage=0) due to the reduced flexibility of OTs that derives from not using BIM, the GA must work more to reach the final optimal solutions and, for this reason, the number of generation is greater.

For greater values of BIM usage, (BIM usage=1) due to the great flexibility of OTs that derives from the full usage of BIM in all its possible applications, the GA must work less to reach the final optimal solutions and, for this reason, the number of generation is lesser.

The above quantitative results demonstrate how BIM represents a flexible and powerful tool, from the cost/benefit point of view, for safety and security management within a proper integrated management model as the considered one [15].

\section{CONCLUSIONS}

The role of BIM for safety and security management for any kind of organization has been illustrated and studied, starting from an already existing management model.

It has also been illustrated its fundamental role in the realization of an already studied Integrated Multidisciplinary Model for Safety and Security management (IMMSSM) which can be supported by a proper Integrated Technological System Framework based on Internet of Everything (IoE-ITSF).

The obtained results have demonstrated the significant improvement of the performances which derives from the use of BIM itself even from the computation time necessary to find the final optimal solution.

In particular way, BIM flexibility and power from the cost/benefit point of view has been demonstrated from the quantitative point of view reaching very interesting values.

\section{REFERENCES}

[1] Zhang, S., Teizer, J., Lee, J.K., Eastman, C.M. \& Venugopal, M., Building information modelling (BIM) and safety: automatic safety checking of construction models and schedules. Automation in Construction, 29, pp. 183-195, 2013. https://doi.org/10.1016/j.autcon.2012.05.006

[2] Zhang, J.P. \& Hu, Z.Z., BIM and 4D-based integrated solution of analysis and management for conflicts and structural safety during construction: 1. principles and methodologies. Automation in Construction, 20(2), pp. 155-166, 2011. https://doi.org/10.1016/j.autcon.2010.09.013

[3] Lu., W., Huang, G. Q. \& Li, H., Scenarios for applying RFID technology in construction project management. Automation in Construction, 20(2), pp. 101-106, 2011.

https://doi.org/10.1016/j.autcon.2010.09.007 
[4] Hensworth, S., Building information modeling and security design. The Australian Building Service Journal, 2, pp. 44-45, 2011.

[5] Porter, S., Terence, T., Tan, T. \& West, G., Breaking into BIM: performing static and dynamic security analysis with the aid of BIM. Automation in Construction, 40, pp. 84-95, 2014.

https://doi.org/10.1016/j.autcon.2013.12.002

[6] Wang, Y., Wang, X., Wang, J., Yung. P. \& Jun, G., Engagement of facilities management in design stage through BIM: framework and a case study. Advances in Civil Engineering, Article ID 189105, 2013.

[7] Ik-Soon, K. \& Hyun-Shik, S., A study on development of intelligent CCTV security system based on BIM. The Journal of the Korea Institute of Electronic Communications Sciences, 6(5), pp. 789-795, 2011.

[8] Garzia, F., Sammarco, E. \& Cusani, R., The integrated security system of the Vatican City State. International Journal of Safety \& Security Engineering, 1(1), pp. 1-17, 2011. https://doi.org/10.2495/safe-v1-n1-1-17

[9] Contardi, G., Garzia, F. \& Cusani, R., The integrated security system of the Senate of the Italian Republic. International Journal of Safety \& Security Engineering, 1(3), pp. 219-246, 2011.

[10] Garzia, F. \& Cusani, R., The integrated safety / security / communication system of the Gran Sasso mountain in Italy. International Journal of Safety \& Security Engineering, 2(1), pp. 13-39, 2012. https://doi.org/10.2495/safe-v2-n1-13-39

[11] Garzia, F. \& Cusani, R., The safety/security/communication wireless LAN of the underground Gran Sasso mountain national laboratories of the Italian Institute of Nuclear Physics. International Journal of Safety \& Security Engineering, 2(3), pp. 209-226, 2012. https://doi.org/10.2495/safe-v2-n3-209-226

[12] Garzia, F., Sammarco, E. \& Cusani, R., Vehicle/people access control system for security management in ports. International Journal of Safety \& Security Engineering, 2(4), pp. 351-367, 2012.

https://doi.org/10.2495/safe-v2-n4-351-367

[13] Garzia, F. \& Papi, L., An internet of everything based integrated security system for smart archaeological areas. Proceedings of the Fifty Annual IEEE International Carnahan Conference on Security Technology, Orlando, Florida, pp. 64-71, 2016.

[14] Garzia, F. \& Sant'Andrea, L., The internet of everything based integrated security system of World War I commemorative museum of Fogliano Redipuglia in Italy. Proceedings of the Fifty Annual IEEE International Carnahan Conference on Security Technology, Orlando, Florida, pp. 56-63, 2016.

[15] Garzia, F., An integrated multidisciplinary model for security management - optimized implementation technique and related supporting technological system framework. Proceedings of the Fifty Annual IEEE International Carnahan Conference on Security Technology, Orlando, Florida, pp. 107-114, 2016.

[16] Lombardi, M., Guarascio, M. \& Rossi, G., The management of uncertainty: model for evaluation of human error probability in railway system. American Journal of Applied Sciences, 11(3), pp. 381-390, 2013.

https://doi.org/10.3844/ajassp.2014.381.390

[17] Guarascio, M., Lombardi, M., Rossi, G. \& Sciarra, G., Risk analysis and acceptability criteria. WIT Transactions on the Built Environment, 94, pp. 131-138, 2007. 
[18] Guarascio, M., Lombardi, M. \& Massi, F., Risk analysis in handling and storage of petroleum products. American Journal of Applied Sciences, 10(9), pp. 965-978, 2013. https://doi.org/10.3844/ajassp.2013.965.978

[19] Broder, J. F. \& Tucker, E., Risk analysis and the security survey. Buttherwoth-Heinemann, New York, 2012.

[20] Garzia, F., Handbook of communication security, WIT Press, Ashurst, 2013.

[21] Borghini, F., Garzia, F., Borghini, A. \& Borghini, G., The psychology of security, emergency and risk. WIT Press, Ashurst, 2016. 